\title{
artigo
}

Melo, L.P.L.; Penha, J.S.; Sardinha, A.H.L.; Coutinho, N.P.S.; Pascoal, L.M.; Ferreira, A.G.N.

Orientações educativas de enfermagem na alta hospitalar de paciente neurosequelado: relato de experiência

\section{Orientações educativas de enfermagem na alta hospitalar de paciente neurosequelado: relato de experiência}

\author{
Education guidelines for nursing in neurosischelled patient hospital: a experience report \\ Directrices educativas para enfermería en neurosis hospital de pacientes escalados: informe de experiencia
}

\begin{abstract}
RESUMO
Objetivo: Descrever a experiência com as orientações educativas de alta hospitalar sob a perspectiva de uma enfermeira inserida no modelo Primary Nursing. Método: Relato desenvolvido a partir da vivência profissional em uma clínica médica de unidade hospitalar em São Luís, Maranhão, de janeiro a dezembro de 2020. As orientações educativas de enfermagem ocorriam através de treinamentos com os cuidadores e familiares dos pacientes, previamente à alta. Resultados: As informações eram repassadas de forma dialogada, priorizando o familiar e/ou cuidador que seria mais presente no domicílio, enfatizando orientações educativas de enfermagem relacionadas aos cuidados gerais com a pele, cuidados com a traqueostomia, gastrostomia ou jejunostomia, administração de dieta enteral e de medicamentos. Conclusão: 0 planejamento das orientações educativas na alta hospitalar são atribuições importantes do enfermeiro primário, promovendo a continuidade do cuidado e segurança por parte dos cuidadores em realizar assistência aos pacientes neurosequelados no ambiente domiciliar.
\end{abstract}

DESCRITORES: Alta do paciente; Educação em Saúde; Enfermagem Primária; Acidente Vascular Cerebral.

\section{ABSTRACT}

Objective: To describe the experience with educational guidelines for hospital discharge from the perspective of a nurse inserted in the Primary Nursing model. Method: Report developed from professional experience in a medical clinic in a hospital in São Luís, Maranhão, from January to December 2020. Educational nursing guidelines occurred through training with caregivers and family members of patients, prior to discharge. Results: The information was passed on in a dialogical manner, prioritizing the family member and / or caregiver who would be most present at home, emphasizing educational nursing guidelines related to general skin care, tracheostomy care, gastrostomy or jejunostomy, administration of enteral diet and medicines. Conclusion: The planning of educational guidelines at hospital discharge are important duties of the primary nurse, promoting continuity of care and safety on the part of caregivers in providing assistance to neuroskilled patients in the home environment.

DESCRIPTORS: Patient Discharge; Health education; Primary Nursing; Stroke.

\section{RESUMEN}

Objetivo: Describir la experiencia con guías educativas para el alta hospitalaria desde la perspectiva de una enfermera insertada en el modelo de Enfermería Primaria. Método: Informe elaborado a partir de la experiencia profesional en una clínica médica en un hospital de São Luís, Maranhão, de enero a diciembre de 2020. Las pautas educativas de enfermería se dieron a través de la capacitación con los cuidadores y familiares de los pacientes, antes del alta. Resultados: La información se transmitió en un diálogo, priorizando al familiar y / o cuidador que estaría más presente en el hogar, enfatizando las pautas educativas de enfermería relacionadas con el cuidado general de la piel, el cuidado de la traqueotomía, la gastrostomía o yeyunostomía, la administración de dieta enteral y medicamentos. Conclusión: La planificación de las pautas educativas al alta hospitalaria son deberes importantes del enfermero primario, promoviendo la continuidad asistencial y la seguridad por parte de los cuidadores en la atención a los pacientes neurocapacitados en el ámbito domiciliário.

DESCRIPTORES: Alta del paciente; Educación en Salud; Enfermería Primaria; Accidente cerebrovascular.

RECEBIDO EM: 28/01/2021 APROVADO EM: 11/02/2021

\section{Liana Priscilla Lima de Melo}

Enfermeira. Especialista em Clínica Médica e Cirúrgica, UFMA. Mestranda do Programa de Pós-graduação em Enfermagem da UFMA.

ORCID: 0000-0002-0263-1119 


\section{Jaiza Sousa Penha}

Enfermeira. Especialista em Saúde da Mulher, UFMA. Mestranda do Programa de Pós-graduação em Enfermagem da UFMA. ORCID: 0000-0001-9805-3802

\section{Ana Hélia de Lima Sardinha}

Enfermeira. Profa. Dra. do Programa de Pós-graduação em Enfermagem da UFMA. ORCID: 0000-0002-8720-6348

\section{Nair Portela Silva Coutinho}

Enfermeira. Profa. Dra. do Programa de Pós-graduação em Enfermagem da UFMA. ORCID: 0000-0002-2050-026X

\section{Lívia Maia Pascoal}

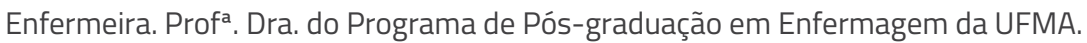
ORCID: 0000-0003-0876-3996

\section{Adriana Gomes Nogueira Ferreira}

Enfermeira. Profa ${ }^{a}$. Dra. do Programa de Pós-graduação em Enfermagem da UFMA. ORCID: 0000-0002-7107-1151

\section{INTRODUÇÃO}

A $s$ doenças cerebrovasculares ocupam o segundo lugar dentre aquelas que ocasionam mais óbitos no mundo, perdendo apenas para as patologias cardiovasculares. Dentre essas doenças a mais frequente é o Acidente Vascular Cerebral (AVC), o qual é considerado um problema de saúde pública, com alta prevalência e alto índice de morbimortalidade, além de estar relacionado ao desenvolvimento de sinais clínicos como distúrbios focais e/ou globais que provocam alterações cognitivas e sensório-motoras ${ }^{1}$.

As sequelas decorrentes de um AVC variam conforme o subtipo da doença, local da obstrução e extensão do tecido cerebral atingido, podendo provocar hemiparesia; hemiplegia; alterações visuais; disartria; disfagia; déficit da memória e alterações emocionais como irritabilidade, esquecimento, confusão e ansiedade ${ }^{2,3}$.

Dessa forma, a demanda de cuidados exigida pelo paciente acometido por AVC é extensa e complexa, devendo ser realizada em instituição de saúde, por uma equipe multiprofissional. Após a recuperação e alta hospitalar, esses cuidados devem ser mantidos no domicílio, por um cuidador ${ }^{4}$. Geralmente, esse cuidador é representado por um familiar de primeiro grau, de sexo feminino, com pouca ou nenhuma formação em saúde, que necessita ser orientado pela equipe para desenvolvê-lo de maneira adequada 5 .

Portanto, a alta hospitalar se caracteriza como uma etapa do cuidado, que deve ser organizada de forma antecipada, pois consiste em uma transição para a continuidade dos cuidados em domicílio 6 . É o momento em que se utiliza do processo de educação em saúde envolvendo paciente e família, compartilhando informações de maneira efetiva, com diferentes recursos de comunicação, certificando-se de que as dúvidas e os problemas foram esclarecidos e solucionados ${ }^{7}$.

Além disso, deve ser individualizada e personalizada, a fim de instruir pacientes e familiares, promovendo a participação da equipe multiprofissional, o auxílio e incentivo ao autocuidado, autoconfiança e autogestão, de forma humanizada e em ambiente acolhedor, facilitando o entendimento das orientações ${ }^{8,9}$.

Assim, revela-se a importância do profissional enfermeiro nesse contexto, em sua rotina como educador, transmitindo conteúdos, ensinando técnicas, explicando, observando e avaliando a prática e a habilidade por parte do cuidador em relação aos procedimentos. Portanto, faz-se necessário que o enfermeiro assuma o papel de coordenar a alta, integrando os demais membros da equipe, oferecendo ao paciente e seus familiares a garantia de um cuidado domiciliar seguro, com qualidade e que preserve a sua autonomia ${ }^{10}$.
Esse papel também está entre as atribuições do enfermeiro primário (ou enfermeiro de referência) inserido no modelo assistencial Primary Nursing (PN), que surgiu em meados de 1968 e foi descrito em 1970, na Universidade de Minessota, por Marie Manthey. $\mathrm{O}$ modelo foi desenvolvido com o objetivo de garantir cuidados de enfermagem completos, individualizados e contínuos, realizados por um enfermeiro primário, que assume o gerenciamento do cuidado disponibilizado a determinados pacientes durante todo o período de internação ${ }^{11}$.

Dentre as vantagens, o $\mathrm{PN}$ permite a aproximação do profissional ao paciente e sua família, favorecendo a autonomia por meio do cuidado humanizado e personalizado $^{12}$. Além disso, esse modelo minimiza a fragmentação da assistência prestada e potencializa a capacidade de liderança, baseada em conhecimento técnico-científico ${ }^{13}$.

Nesse sentido, considerando-se que o planejamento da alta é um processo complexo, com início ainda na admissão do paciente, onde são coletadas informações importantes, como os sistemas de apoio familiar e o ambiente doméstico, o enfermeiro primário tem papel fundamental nesta fase da internação hospitalar ${ }^{14}$. Ao estabelecer vínculo com o paciente e a família, por acompanhar todo o processo de internação, esse profissional tem a capacidade de identificar as habilidades do cuidador, fornecendo segurança através de orientações em saú- 
de voltadas para o domicílio, baseadas em evidências científicas, que poderão permitir a continuação do cuidado ${ }^{15}$.

Diante do exposto, obtém-se a seguinte pergunta norteadora: quais as orientações educativas de enfermagem voltadas para a alta hospitalar de pacientes neurosequelados?

Este estudo tem o objetivo de descrever a experiência de uma enfermeira inserida no modelo assistencial Primary Nursing com as orientações educativas de alta hospitalar.

\section{MÉTODOS}

Estudo descritivo, do tipo relato de experiência, sobre a vivência das orientações de enfermagem voltadas para a alta hospitalar de paciente neurosequelado, realizadas por uma enfermeira da clínica médica, em um hospital de São Luís, no estado do Maranhão, de janeiro a dezembro de 2020. A profissional possui seis anos de formação, com pós-graduação em Clínica Médica e Cirúrgica e Enfermagem em Estomaterapia, atuando como enfermeira primária na instituição há quatro anos.

A referida instituição hospitalar oferece atendimento exclusivo para servidores públicos estaduais e possui uma unidade de clínica médica que adota o modelo assistencial Primary Nursing desde 2017, dispondo de 39 leitos. O perfil da clientela assistida consiste, em sua maioria, de idosos e pacientes portadores de doenças crônicas como: cardiopatas, nefropatas, portadores de pé diabético e neurosequelados, principalmente após AVC.

As orientações educativas de enfermagem eram planejadas e ocorriam através de treinamentos com os cuidadores e familiares dos pacientes, previamente à alta, e priorizavam quatro temáticas comuns à maioria dos pacientes neurosequelados: cuidados gerais com a pele, cuidados com a traqueostomia, com a gastrostomia ou jejunostomia, administração de dieta enteral e medicamentos. Todas essas informações eram disponibilizadas também de forma escrita às famílias.

Por se tratar de um relato de experiência não houve a necessidade de utilização do Termo de Consentimento Livre e Es- clarecido (TCLE). Foram obedecidos os preceitos éticos da Resolução do Conselho Nacional de Saúde n466/2012 durante este estudo e não houve divulgação de nenhum dado que possibilitasse identificação dos participantes.

\section{RELATO DE EXPERIÊNCIA}

Após ser comunicada da previsão de alta, pelo profissional médico, a enfermeira primária dava início a sua atuação, informando a família sobre essa decisão, seguindo a rotina de orientações. Estas eram realizadas, preferencialmente, uma semana antes da alta hospitalar, na enfermaria, à beira leito do paciente, divididas em dois momentos.

Em todas as orientações, o primeiro momento era teórico explicativo, em uma abordagem dialógica, sobre os cuidados necessários e, em seguida, um momento prático, em que os familiares e/ou cuidadores eram estimulados a manusear os dispositivos invasivos (sonda de gastrostomia, etc.), para que a profissional avaliasse as habilidades e segurança dos cuidadores.

Em relação aos cuidados gerais com a pele, a enfermeira forneceu orientações sobre a utilização de sabonetes adequados para a higiene corporal e hidratantes com função umectante. Também foram reforçadas as orientações sobre a mudança de decúbito a cada duas horas, mesmo em ambiente domiciliar, a fim de prevenir lesões por pressão.

Para os cuidados com a traqueostomia, foram dadas orientações quanto ao modo de limpeza da cânula, metálica ou plástica e a maneira adequada de realizar aspiração traqueal. Já para os cuidados com a gastrostomia e jejunostomia, eram enfatizadas informaçôes principalmente sobre os sinais de complicações, como rubor na pele ao redor, extravasamento de efluente gástrico ou entérico, obstrução e perda acidental deste dispositivo.

Quanto aos aspectos nutricionais dos pacientes, os familiares e cuidadores receberam orientações sobre a importância da higiene das mãos para o preparo da dieta, os tipos de dieta que poderiam ser ofertadas e um treinamento prático sobre o manuseio dos dispositivos de gastrostomia e jejunostomia durante a administração de dietas, medicamentos e verificação de resíduo gástrico.

\section{DISCUSSÃO}

A participação ativa da enfermeira primária no processo de alta dos pacientes foi reforçada pelos resultados de uma revisão sistemática, na qual as evidências sobre a adoção do modelo assistencial Primary Nursing nas instituições de saúde foram satisfatórias, principalmente pelo vínculo estabelecido, em que o enfermeiro primário apoia o enfrentamento dos membros da família do paciente durante os períodos de hospitalização ${ }^{16}$.

Apesar disso, os resultados apresentados neste relato de experiência divergem dos dados encontrados em estudo que objetivou analisar a atuação do enfermeiro no preparo da alta hospitalar de pacientes cirúrgicos. Na referida investigação, as orientações pertinentes à alta foram ausentes, o que contribuiu para a insegurança e ansiedade dos mesmos ${ }^{17}$, destacando a relevância dessas informações para a continuidade dos cuidados.

Com relação ao direcionamento das orientações educativas principalmente aos familiares e cuidadores dos pacientes, identificou-se achado semelhante em estudo com abordagem qualitativa sobre critérios de alta em pacientes neurológicos. Os autores concluíram que o preparo da alta hospitalar para portadores de lesão neurológica incapacitante deve envolver não somente o cliente, mas também a família e pessoas significativas a ele, contribuindo para o processo de reabilitação ${ }^{18}$.

As orientações educativas de enfermagem para alta hospitalar de pacientes neurosequelados foram direcionadas para os quatro principais eixos que merecem atenção especial, corroborando com estudo que objetivou construir um modelo de plano de alta para pacientes hospitalizados após acidente vascular cerebral. Comprovou-se que essas informações são essenciais para que a transferência de responsabilidade do cuidado aconteça de forma segura ${ }^{19}$.

$\mathrm{O}$ uso de recursos didáticos no cuidado 
em saúde promove empoderamento, emancipação de saberes e satisfação de pacientes e familiares em participar do processo educativo, favorecendo autonomia e qualidade de vida20. No entanto, identificou-se a ausência desses materiais de apoio, além da inexistência de protocolo institucional para realização do procedimento, a fim de nortear a prática dos enfermeiros, não só do enfermeiro primário, o que poderia facilitar e dinamizar esse momento.

\section{CONCLUSÃO}

As orientações educativas na alta hospitalar planejada são atribuições importantes do enfermeiro primário por promover a continuidade do cuidado e fornecer segurança aos cuidadores na realização dessas ações com os pacientes neurosequelados no ambiente domiciliar. Para tanto, é necessário que esses profissionais embasem as suas atividades em evidências científicas atualizadas, garantindo a cientificidade do cuidado de enfermagem.

A padronização de técnicas e recursos materiais educativos podem contribuir para o sucesso da realização desse procedimento. No entanto, há a necessidade da avaliação crítica por parte do enfermeiro e demais profissionais, ao considerar a individualidade e a singularidade do sujeito assistido, contribuindo para a alta hospitalar personalizada.

\section{REFERÊNCIAS}

1. BRASIL. Manual de rotinas para atenção ao AVC / Ministério da Saúde, Secretaria de Atenção à Saúde, Departamento de Atenção Especializada. Brasília: Ed. do Ministério da Saúde, 2013.

2. American Stroke Association. Stroke risk factors. Disponivel em: <https://www.stroke.org/en>. Acesso em: 7 dez. 2020.

3. Silva RCD, Gurian JG, Curi M, Timpone LA, Judice MG, Arantes APF. Functionality and quality of life of stroke survivors after ICU discharge. Rev Inspirar Mov. e Saúde, 2019 19(4):1-18.

4. Nóbrega MF, Nascimento KP, Pinheiro AB. Assistência de enfermagem ao paciente com acidente cerebrovascular: revisão integrativa da literatura brasileira. Braz J. Dev., 2019 5(11):2746227478.

5. Oliveira EC, Ferreira WFS, Oliveira EC, Dutra DA. Post-high care in older patients with vaccine vascular dental sequels: high hospital planning. Rev Sau e Desenv, 2017 11(9):172-197.

6. Cieto BB, Garbuio DC, Camargo VB, Napoleão AA. Recursos e inovações de enfermagem para a alta: revisão integrativa. Rev Min Enf, 2014 18(3):752-757. DOI: 10.5935/1415-2762.20140055.

7. Guzinski, C, Lopes ANM, Flor J, Migliavaca J, Tortato C, Dal Pai D. Boas práticas para comunicação efetiva: a experiência do round interdisciplinar em cirurgia ortopédica. Rev Gaúcha Enferm, 2019;40(esp):e20180353. DOl: https://doi.org/10.1590/19831447.2019.20180353.

8. Fontana G, Chesani FH, Menezes M. As significações dos profissionais da saúde sobre o processo de alta hospitalar. Saúde Transform. Soc., 2017 8(2):86-95.

9. Wachholz LF, Knihs NS, Martins SR, Magalhães ALP, Brehmer LCF, Martins MS. Alta hospitalar do paciente transplantado hepático: revisão integrativa. Esc. Anna Nery, 2020 24(4):1-9. DOI: DOI: 10.1590/2177-9465-EAN-2019-0346.

10. Góes FGB, Cabral IE. Discourses on discharge care for children with special healthcare needs. Rev. Bras. Enferm. [Internet]. 2017;70(1):154-61. DOI: 10.1590/0034-7167-2016-0248

11. Sellick KJ, Russell S, Beckmann JL. Primary nursing: an evaluation of its effects on patient perception of care and staff satisfaction. Int. J. Nurs. Stud. 2003 40(5): 545-551. DOI: 10.1016/ S0020-7489(03)00064-6.

12. Andrade D, Alves MB, Gobo DC, Silva EA, Baia WRM. Im- plantação do Modelo Enfermeiro Referência "Primary Nursing" em um Hospital Público Oncológico no Estado de São Paulo. In: Anais do Congresso Internacional de Humanidades \& Humanização em Saúde; 2014, 2(1). São Paulo: Editora Blucher, 2014. ISSN 2357-7282 DOI 10.5151/medpro-cihhs-10314.

13. Santos KCB dos, Cavalcante TB, Ribeiro ASFM de. Implantação do modelo enfermeiro de referência em um hospital universitário. Rev. Enferm. UFPE Online., 2019 13(1):268-74. DOI: 10.5205/1981-8963-v13i01a236542p268-274-2019.

14. Mennuni M, Gulizia MM, Alunni G, Amico AF, Bovenzi FM, Caporale $\mathrm{R}$ et al. ANMCO position paper: hospital discharge planning: recommendations and standards. Eur. Heart J. 2017,19(2):244-255. DOI: 10.1093/eurheartj/sux011.

15. Sousa ETG, Maia DB, Neto WZA, Costa MCR, Gama RM, Gomes LFR. Preparation for the Hospital Discharge of Neurosurgical Patients and their relatives: experience report. J Nurs UFPE Online. 2014, 8(1):207-2012. DOI: 10.5205/reuol.4843-395941-SM.0801201429.

16. Matilla $E$, Pitkanen A, Leino $K$, Luojus $K$ et al. The Effects of the Primary Nursing Care Model: A Systematic Review. J. Nurs. Care 2014, 3(6): 1-12. DOI: 10.4172/2167-1168.1000205.

17. Martins KP, Costa KNFM, Oliveira DST, Valdevino SC, Rezende LCM et al. Atuação do enfermeiro no preparo para a alta hospitalar de pacientes cirúrgicos Rev. Pes. Cuidado é Fundamental Online. 2015, 7(1): 1756-1764. DOI: 10.9789/2175-5361.2015. v7i1.1756-1764.

18. Machado WCA, Silva VM, Silva RA, Ramos RL, Figueiredo NMA et al. Alta hospitalar de clientes com lesão neurológica incapacitante: impreteríveis encaminhamentos para reabilitação. Cienc. e Saúde Coletiva 2016, 21(10): 3161-3170. DOI: 10.1590/1413-812320152110.17232016.

19. Moro CK. Plano de alta hospitalar para pacientes após acidente vascular cerebral. [Dissertação] Programa de Pós-Graduação em Enfermagem: Universidade do Vale do Rio dos Sinos; 2019.

20. Pereira ELC, Sanguino GZ, Ronchi TS, et al. Tecnologias Educativas Gerontogeriátricas nas Diferentes Temáticas de Saúde: Uma Revisão Integrativa. Rev. Enfer. Centro Oeste Min. 2019;9:e2768. DOI: 10.19175/recom.v9i0.2768 